\title{
Influence of the Urban Exposome on Birth Weight
}

\author{
Mark J. Nieuwenhuijsen, ${ }^{1,2,3 *}$ Lydiane Agier, ${ }^{4 *}$ Xavier Basagaña, ${ }^{1,2,3}$ Jose Urquiza, ${ }^{1,2,3}$ Ibon Tamayo-Uria,5 \\ Lise Giorgis-Allemand, ${ }^{4}$ Oliver Robinson, ${ }^{6}$ Valérie Siroux, ${ }^{4}$ Léa Maitre, ${ }^{1,2,3}$ Montserrat de Castro, ${ }^{1,2,3}$ Antonia Valentin, ${ }^{1,2,3}$ \\ David Donaire, ${ }^{1,2,3}$ Payam Dadvand, ${ }^{1,2,3}$ Gunn Marit Aasvang, ${ }^{7}$ Norun Hjertager Krog, ${ }^{7}$ Per E. Schwarze, ${ }^{7}$ Leda Chatzi, ${ }^{6,8,9,10}$ \\ Regina Grazuleviciene, ${ }^{11}$ Sandra Andrusaityte, ${ }^{11}$ Audrius Dedele, ${ }^{11}$ Rosie McEachan, ${ }^{12}$ John Wright, ${ }^{12}$ Jane West, ${ }^{12}$ \\ Jesús Ibarluzea,,$^{3,13,14,15}$ Ferran Ballester, ${ }^{2,3,16,17}$ Martine Vrijheid, ${ }^{1,2,3}$ and Rémy Slama ${ }^{4}$
}

${ }^{1}$ ISGlobal (Institute for Global Health), Barcelona, Spain

${ }^{2}$ Universitat Pompeu Fabra (UPF), Barcelona, Spain

${ }^{3}$ CIBER Epidemiología y Salud Pública (CIBERESP), Madrid, Spain

${ }^{4}$ Team of environmental epidemiology applied to reproduction and respiratory health, Institut national de la santé et de la recherche médicale (Inserm, National Institute of Health \& Medical Research), Institute for Advanced Biosciences (IAB), CNRS, Université Grenoble Alpes, Grenoble, France

${ }^{5}$ Department of Statistics, Faculty of Arts and Sciences, Harvard University, Cambridge, Massachusetts, USA

${ }^{6}$ MRC-PHE Centre for Environment and Health, School of Public Health, Imperial College London, London, UK

${ }^{7}$ Norwegian Institute of Public Health (NIPH), Oslo, Norway

${ }^{8}$ Department of Preventive Medicine, Keck School of Medicine, University of Southern California, Los Angeles, USA

${ }^{9}$ Department of Social Medicine, University of Crete, Greece

${ }^{10}$ Department of Genetics \& Cell Biology, Faculty of Health, Medicine and Life Sciences, Maastricht University, Maastricht, Netherlands

${ }^{11}$ Vytauto Didžiojo Universitetas, Kaunas, Lithuania

${ }^{12}$ Bradford Institute for Health Research Bradford, Bradford, UK

${ }^{13}$ Faculty of Psychology, University of the Basque Country UPV/EHU, San Sebastian, Basque Country, Spain

${ }^{14}$ Health Research Institute, BIODONOSTIA, San Sebastian, Basque Country, Spain

${ }^{15}$ Sub-Directorate for Public Health of Gipuzkoa, Department of Health, Government of the Basque Country, San Sebastian, Basque Country, Spain

${ }^{16}$ Nursing School, Universitat de València, Valencia, Spain

${ }^{17}$ Joint Research Unit of Epidemiology and Environmental Health, FISABIO-Universitat Jaume I-Universitat de València, Valencia, Spain

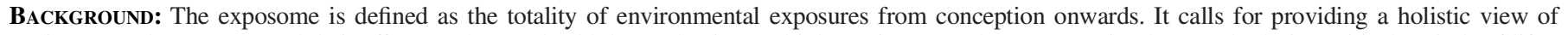
environmental exposures and their effects on human health by evaluating multiple environmental exposures simultaneously during critical periods of life.

ОвJеCTIVE: We evaluated the association of the urban exposome with birth weight.

Methods: We estimated exposure to the urban exposome, including the built environment, air pollution, road traffic noise, meteorology, natural space, and road traffic (corresponding to 24 environmental indicators and 60 exposures) for nearly 32,000 pregnant women from six European birth cohorts. To evaluate associations with either continuous birth weight or term low birth weight (TLBW) risk, we primarily relied on the DeletionSubstitution-Addition (DSA) algorithm, which is an extension of the stepwise variable selection method. Second, we used an exposure-by-

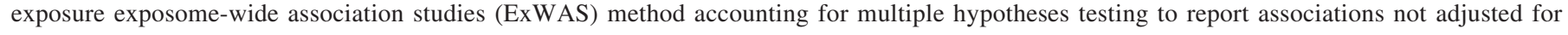
coexposures.

RESULTS: The most consistent statistically significant associations were observed between increasing green space exposure estimated as Normalized Difference Vegetation Index (NDVI) and increased birth weight and decreased TLBW risk. Furthermore, we observed statistically significant associations among presence of public bus line, land use Shannon's Evenness Index, and traffic density and birth weight in our DSA analysis.

ConCLUSION: This investigation is the first large urban exposome study of birth weight that tests many environmental urban exposures. It confirmed previously reported associations for NDVI and generated new hypotheses for a number of built-environment exposures. https://doi.org/10.1289/EHP3971

\section{Introduction}

The exposome is defined as the totality of environmental exposures from conception onwards (Wild 2005, 2012). This defini-

\footnotetext{
*Both authors are joint first author.

Address correspondence to Mark J. Nieuwenhuijsen, PhD, Research Professor in Environmental Epidemiology, Director Air Pollution and Urban Environment, Director Urban Planning, Environment and Health Initiative, ISGlobal, Barcelona Institute for Global Health - Campus MAR, Barcelona Biomedical Research Park (PRBB), Doctor Aiguader, 88, 08003 Barcelona, Spain. Telephone: +34 932147337 । +34 6080508 14. Email: Mark. nieuwenhuijsen@isglobal.org Skype: Mark.nieuwenhuijsen

Supplemental Material is available online (https://doi.org/10.1289/EHP3971).

The authors declare they have no actual or potential competing financial interests.

Received 30 May 2018; Revised 20 February 2019; Accepted 7 March 2019; Published 22 April 2019.

Note to readers with disabilities: $E H P$ strives to ensure that all journal content is accessible to all readers. However, some figures and Supplemental Material published in EHP articles may not conform to 508 standards due to the complexity of the information being presented. If you need assistance accessing journal content, please contact ehponline@ niehs.nih.gov. Our staff will work with you to assess and meet your accessibility needs within 3 working days.
}

tion aims to provide a holistic view of environmental exposures and their effects on human health by evaluating multiple exposures simultaneously during critical time periods of life. Over recent years, there has been considerable discussion on the topic (Lioy and Rappaport 2011; Siroux et al. 2016; Slama and Vrijheid 2015; Stingone et al. 2017; Wild 2012), but little actual work has been conducted to put the exposome concept into practice. Challenges include capturing the breadth of and variability in environmental exposures, which entails hundreds or more exposure variables (Robinson et al. 2015), and statistical challenges such as efficiency of variable selection techniques in the presence of numerous correlated covariates (Agier et al. 2016).

Until now, exposome studies have been conducted mainly on routinely collected or cross-sectional datasets, such as those examining environmental risk factors for type 2 diabetes (Patel et al. 2010), serum lipids (Tzoulaki et al. 2012), all causes mortality (Patel et al. 2013b), pre-term birth (Patel et al. 2013a), metabolic syndrome (Lind et al. 2013), and telomere length (Patel et al. 2016). No studies have been conducted on birth weight.

Previous studies have shown negative associations between birth weight and environmental exposures such as air pollution, noise, temperature or lack of green space (Dadvand et al. 2012; Dzhambov et al. 2014; Hohmann et al. 2013; Nieuwenhuijsen et al. 
2013; Pedersen et al. 2013; Zhang et al. 2017). Recent reviews suggested that there was sufficient evidence for a relationship between air pollution and impaired fetal growth; limited evidence for a relationship between green space and fetal growth; and inadequate evidence for a relationship between noise and extreme temperatures and fetal growth, respectively (Gascon et al. 2016; Vrijheid et al. 2016). Gascon et al. (2016) found no studies on fetal growth and built environment measures such as connectivity, density, and walkability. Two recent studies reported an association between disadvantaged neighborhoods and adverse birth outcomes (Ncube et al. 2016; Vos et al. 2014). With some exceptions (Dadvand et al. 2014; Hystad et al. 2014), generally these exposures have been evaluated individually or considering single exposure families (in the case of atmospheric pollutants), i.e., one exposure or exposure family at a time, thus not accounting for possible confounding effects of coexposures outside the family.

The Human Early Life Exposome (HELIX) is one of the first large exposome projects. It aims to characterize early life exposure to multiple environmental factors and associate these with child health outcomes (Vrijheid et al. 2014). The project uses harmonized data from six existing birth cohort studies with $\sim 32,000$ mother child pairs in Europe and includes a new followup with in-depth examinations of a subset of children (Maitre et al. 2018). Novel tools and methods were implemented to characterize environmental exposures, e.g., remote sensing, personal sensing in a subpopulation, and GIS-based spatial methods (Robinson et al. 2018).

In this study, we aimed to evaluate the association of the urban exposome, which includes the built environment, air pollution, road traffic noise, meteorology, natural space, and road traffic indicators (60 exposures covering 24 environmental indicators) with birth weight and term low birth weight (TLBW). For our main analysis, we used the Deletion-Substitution-Addition (DSA) algorithm, a variable selection method that partly accounts for potential confounding by coexposures; we initially showed in a simulation study that this method provided the best performance to identify the truly (causally) associated exposures in an exposome context. We additionally applied an exposure-by-exposure exposome-wide association study (ExWAS) to test for and show possible exposure-health associations, and to screen for additional coexposure confounding factors.

\section{Method}

\section{Study Population}

The study population comprises 31,458 singleton pregnancies from six European birth cohorts based in nine cities; Born in Bradford (BiB; Bradford, UK) (Wright et al. 2013), Étude des Déterminants pré et postnatals du développement et de la santé de l'Enfant (EDEN; Poitiers and Nancy, France) (Drouillet et al. 2009), INfancia y Medio Ambiente (INMA; Gipuzkoa, Sabadell, and Valencia, Spain) (Guxens et al. 2012), Kaunus Cohort (KANCLithuania; Kaunas, Lithuania) (Grazuleviciene et al. 2009), Norwegian Mother and Child Cohort Study (MoBa; Norway) (Magnus et al. 2006), and Rhea Study (Mother and Child Cohort in Crete, Greece) (Chatzi et al. 2017).

\section{Health Outcome and Adjustment Factors}

We considered two health outcomes: birth weight (in grams) and TLBW, defined as weight $<2500$ grams at birth after 37 wk of gestation. We adjusted all analyses for a (fixed) city effect, for gestational age (calculated from date of last menstruation, or ultrasoundbased, values not rounded; simple and quadratic terms), sex of the newborn, parity, maternal height and weight [using a broken stick model with a knot at $60 \mathrm{~kg}$ (Pedersen et al. 2013)] before pregnancy, mean number of cigarettes smoked per day by the mother during the second trimester of gestation, maternal education (as the main social class confounder), and season of conception.

\section{Characterization of the Urban Exposome}

An extensive assessment of the urban exposome was conducted using a geographic information system (GIS)-based environmental model built for the whole HELIX study area and assigning exposures to pregnancy home addresses. The detailed description of the exposure assessment can be found in Robinson et al. (Robinson et al. 2018). For EDEN, BiB, and KANC, address information was available at the time of recruitment, whereas for INMA, Rhea, and MoBa, address information was available at the time of birth of the child.

For each woman, assessment of exposure at the residential address during pregnancy was made within PostgreSQL ${ }^{\mathrm{TM}}$ (copyright (C) 1996-2017 The PostgreSQL Global Development Group) and PostGIS platforms for all groups of environmental factors but road traffic.

Topological maps for built environment were obtained from local authorities or from Europe-wide sources. Building density was calculated within $100 \mathrm{~m}$ and $300 \mathrm{~m}$ buffers by dividing the area of building cover $\left(\mathrm{m}^{2}\right)$ by the area of buffer $\left(\mathrm{km}^{2}\right)$. Connectivity density was calculated as the number of street intersections inside $100 \mathrm{~m}$ and $300 \mathrm{~m}$ buffers, divided by the area $\left(\mathrm{km}^{2}\right)$ of each buffer. Land use Shannon's Evenness Index (SEI) was calculated as the proportional abundance of each land use type multiplied by that proportion, divided by the logarithm of the number of land use types, in a buffer of $300 \mathrm{~m}$, giving a score of 0 to 1 . Bus public transport (BST) lines and stops were obtained from local authorities of each study area and from Open Street Maps ("OpenStreetMap") where local layers were not available. The presence of public bus lines was assessed within 100-m, 300-m, and 500-m buffers. The density of public bus stops was calculated as the number of BST inside 100-m, 300-m, and 500-m buffers, divided by the buffer area. Facility density was calculated as the number of facilities present divided by the area of the 300-m buffer. Facility richness index was calculated as the number of different facility types present divided by the maximum potential number of facility types specified, in a buffer of $300 \mathrm{~m}$, giving a score of 0 to 1 . Population density was calculated as the number of inhabitants per square kilometer at the home address (from $100 \mathrm{~m} \times 100 \mathrm{~m}$ raster). We developed an indicator of walkability, adapted from the previous walkability indices (Duncan et al. 2014; Frank et al. 2006), calculated as the mean of the deciles of population density, street connectivity, facility richness index, and land use SEI within 300-m buffers, giving a walkability score ranging from 0 to 1 .

For the assessment of air pollutants, including particulate matter (PM) with an aerodynamic diameter of less than $2.5 \mu \mathrm{m}$ $\left(\mathrm{PM}_{2.5}\right)$ and of less than $10 \mu \mathrm{m}\left(\mathrm{PM}_{10}\right)$, absorbance of $\mathrm{PM}_{2.5}$ filters and nitrogen dioxide $\left(\mathrm{NO}_{2}\right)$, we used land use regression (LUR) or dispersion models, temporally adjusted to measurements made in local background monitoring stations. For most cities, we used site-specific LUR models developed in the context of the ESCAPE project (Beelen et al. 2013; Eeftens et al. 2012). In $\mathrm{BiB}$, assessment for $\mathrm{PM}_{2.5}$ and $\mathrm{PM}_{10}$ was made based on the ESCAPE LUR model developed in the Thames Valley region of the United Kingdom and adjusted for background PM levels from monitoring stations in Bradford, UK (Schembari et al. 2015). The ESCAPE European-wide LUR model was applied for $\mathrm{PM}_{2.5}$ in Nancy and Poitiers (EDEN) and Gipuzkoa and Valencia (INMA) and corrected for local background monitoring data 
(Wang et al. 2014). In Gipuzkoa and Valencia (INMA), $\mathrm{PM}_{10}$ estimates were made based on local ratios to $\mathrm{PM}_{2.5}$ estimates. In Nancy and Poitiers (EDEN), dispersion models were used to assess $\mathrm{NO}_{2}$ and $\mathrm{PM}_{10}$ exposure (Rahmalia et al. 2012). Air pollutants were averaged over each trimester of pregnancy and over the whole pregnancy period.

Road traffic noise levels were derived from noise maps produced in each local municipality under the 2010 European Environmental Noise Directive (European Environment Agency 2010). To improve comparability between centers, we also considered a categorized version of the variables.

Regarding meteorology, daily measurements of temperature and humidity were obtained from a local weather station in each study area. Daily measurements of ultraviolet (UV) radiation at $0.5 \times 0.5$-degree resolution were obtained from the Global Ozone Monitoring Experiment on board the European Remote Sensing (ERS-2) satellite (http://www.temis.nl/uvradiation/archives/). Values were averaged over each trimester of pregnancy and over the whole pregnancy period.

For natural space indicators, we followed the PHENOTYPE protocol (Nieuwenhuijsen et al. 2014) to measure the surrounding vegetation, i.e., trees, shrubs and parkland, and applied the Normalized Difference Vegetation Index (NDVI) (Weier and Herring 2000) derived from the Landsat 4-5 Thematic Mapper (TM) satellite images at $30 \mathrm{~m} \times 30 \mathrm{~m}$ resolution. To achieve maximum exposure contrast, we looked for available cloud-free Landsat TM images during the period between May and August for years relevant to our period of study and calculated greenness within 100-m, 300-m, and 500-m buffers around each address. We calculated access to major green spaces (parks or countryside) and blue spaces (bodies of water) as the straight line distance from the home to nearest blue or green space with an area greater than $5,000 \mathrm{~m}^{2}$ from topographical maps (e.g., Urban Atlas 2006 or local sources).

Road traffic indicators (traffic density on nearest road, traffic load on all roads, presence of traffic on the major road within $100 \mathrm{~m}$, and inverse distance to nearest major road) were calculated from traffic road network maps following the ESCAPE protocol (Beelen et al. 2013).

The exposure assessment methodology is further detailed elsewhere (Robinson et al. 2018), together with the correlation structure (defined by computing the pairwise Pearson, polyserial or polychoric correlation coefficients, as appropriate) and main characteristics by cohort.

We excluded exposures that did not provide enough information, i.e., those that had $>70 \%$ missing values, whose variability was mainly due to between-cities variations (i.e., the ratio of the city-centered data vs. raw data standard deviation was $>30 \%$ ) or that were correlated at a level $>0.99$ in absolute value with another variable (14 exposure variables were excluded; see Table S1). Sixty exposure variables were retained for a total of 24 environmental indicators, covering six fields: the built environment (15 exposures), air pollution (16 exposures), road traffic noise (4 exposures), meteorology (12 exposures), natural space ( 9 exposures), and road traffic (4 exposures). The exposure levels are described in Table 1.

\section{Missing Data Imputation}

The same procedure for missing data imputation was used for all HELIX studies (Robinson et al. 2018). All exposures were transformed to approach normality, using a Box-Cox power transformation approach that chooses among raising the data to the power $-2,-1,-0.5$, one-third, $0.5,1$, or 2 , or logtransforming. Missing data for all adjustment factors and exposures were imputed using the chained equations method; birth weight was not imputed as no gain in power is expected from imputing the explained variable (White et al. 2011). Birth weight and a city indicator were forced in as predictors in the imputation models. Other variables (including postnatal exposures) were used as predictors when they were correlated with the exposure to be imputed [i.e., absolute correlation value in the $(0.4 ; 0.9)$ range] and/or with the probability of the exposure being missing (i.e., absolute correlation value $>0.4$ ), while ensuring their proportion of nonmissing observations among the observations with missing values in the exposure to be imputed was greater than $40 \%$. We restricted the number of predictors in the imputation models to fewer than 25 variables (Van Buuren 2018). Because of the large study population size that limits the potential impact of the imputation procedure, a single dataset was imputed. For all the exposures whose imputed and nonimputed data could be compared, we found that imputed and nonimputed values were comparable. If the exposure was continuous, we computed the average and standard deviation of nonimputed and imputed values altogether and separately after standardization on city averages. We ensured that the absolute difference between means of the nonimputed and imputed values was smaller than 2 standard deviations and that the ratio of standard deviation of the nonimputed values vs. the imputed values was in the 0.5 to 2 range (Stuart et al. 2009); if the exposure was categorical, we ensured the $p$-value of the chi-squared test between imputed and nonimputed values was $>5 \%$.

All continuous exposures were standardized so that an increase by one unit corresponds to the interquartile range (IQR) of the transformed to approach normality exposure values.

\section{Estimating the Exposome-Health Association}

In an exposome context in which a large number of exposures are investigated, variable selection is the first challenge to face when testing association with a health outcome before other targets, such as point estimates or prediction, can be further optimized. Here, we adapted a simulation study (Agier et al. 2016) that compared several statistical methods to assess exposome-health associations, with the aim to identify the most efficient method in terms of differentiating exposures that were or were not associated with the outcome. We tested six agnostic methods: ExWAS, ExWAS followed by multiple linear regression model including all exposures that were selected in ExWAS, or ExWAS-multiple linear regression (ExWAS-MLR), elastic net (ENET), sparse partial least squares (sPLS), graphical unit evolutionary stochastic search (GUESS), and DSA. The initial simulation was performed considering a set of 237 exposures measured in a population of 1,200 individuals (Agier et al. 2016). Here, we generated 57 exposures based on the HELIX urban exposome correlation structure for a total of 32,000 subjects, to estimate the models' performances that could be expected with our study design. We generated a fictitious health outcome that was linearly related to 1 to 25 of these 57 exposures. We applied the statistical methods (except for GUESS which could not be run due to limitation of the $\mathrm{R}$ function implementation) and compared their performances by computing the averaged sensitivity and false discovery proportion (FDP) over 100 simulation runs.

As Agier et al. (2016) observed, we found that, in a context similar to our study design, DSA best accomodates our data structure when performing variable selection, in comparison with other multivariate methods. DSA had an average sensitivity of $99.7 \%$ and an average FDP of $5.7 \%$ (Figure 1, Figure S1 for additional details). DSA is a multivariate regression-based variable selection method that relies on an iterative model search algorithm (Sinisi and van der Laan 2004). It can be seen as an extended and more 
Table 1. Description of factors included in the HELIX prenatal urban exposome study and ExWAS associations of each variable with birth weight and term low birth weight, adjusted for potential confounders.

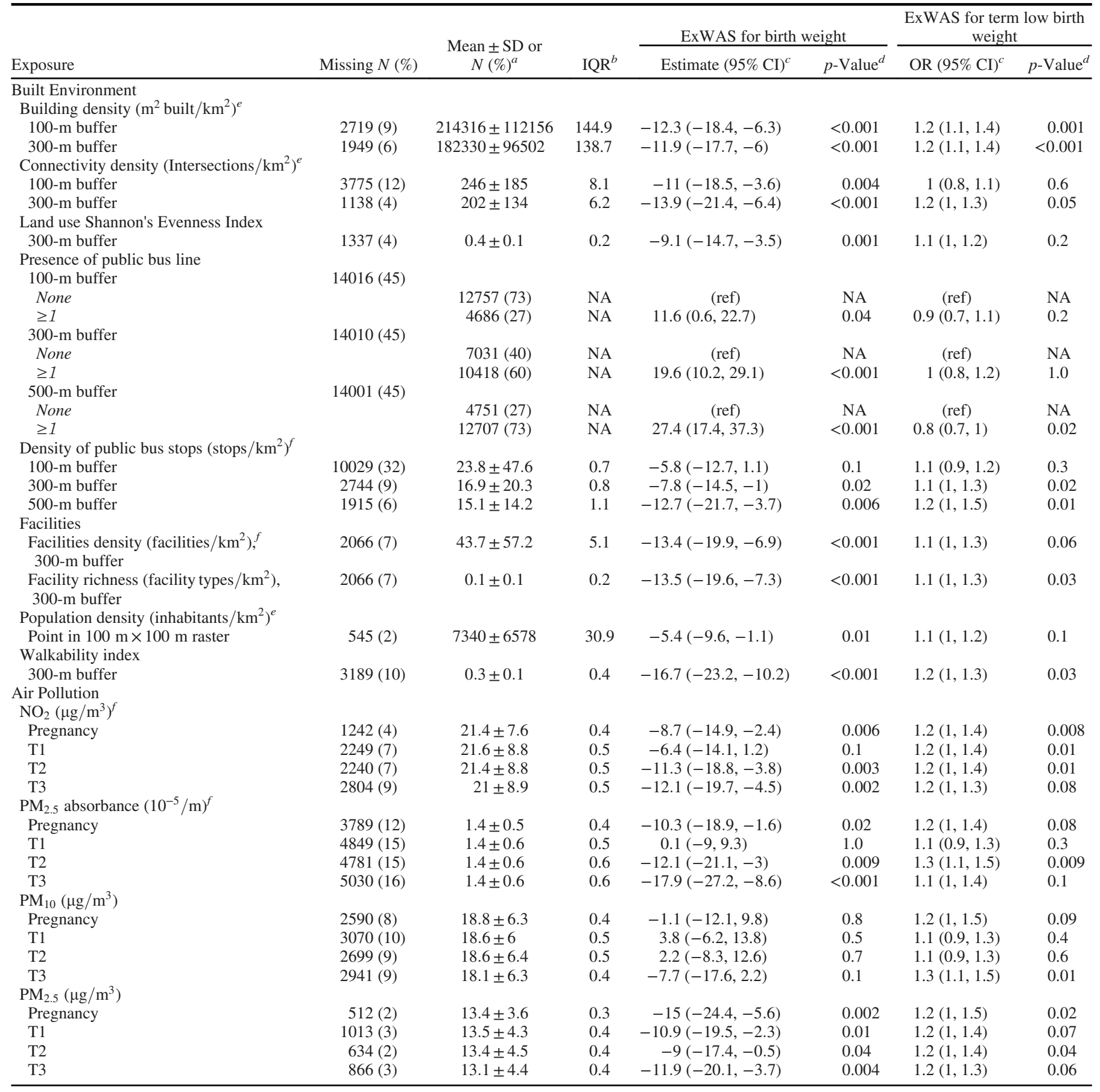

Note: All buffer areas and distances are relative to the pregnancy residence unless otherwise indicated. Missing exposure and covariate data were imputed using chained equations. CI, confidence interval; ExWAS, exposome-wide association study; DNA, deoxyribonucleic acid; IQR, interquartile range; NA, not applicable; NDVI, normalized difference vegetation index; $\mathrm{NO}_{2}$, nitrogen dioxide; OR, odds ratio; $\mathrm{PM}_{2.5}$, particulate matter (PM) with an aerodynamic diameter of less than $2.5 \mu \mathrm{m} ; \mathrm{PM}_{10}$, $\mathrm{PM}$ with an aerodynamic diameter of less than $10 \mu$ m; ref, reference category for binary and categorical exposures; SD, standard deviation; T1, first trimester of pregnancy; T2, second trimester of pregnancy; T3, third trimester of pregnancy. ${ }^{a}$ Distribution is displayed over nonimputed values.

${ }^{b}$ The IQR is computed over continuous exposures only, after imputing missing values and transforming data to approach normality.

${ }^{c}$ Difference in mean birth weight or odds ratio (OR) for term low birth weight in association with an IQR increase in (imputed and transformed to approach normality) exposure (continuous variables), or relative to the reference category (binary and categorical variables), adjusted for a city effect, gestational age (simple and quadratic terms), sex of the newborn, parity, maternal height and weight before pregnancy, maternal smoking, maternal education and season of conception.

${ }^{d}$ Uncorrected $p$-values testing the global significance of the variable, obtained using a Wald test (continuous or binary variables) or a likelihood ratio test (categorical noise variables). Multiple hypothesis testing corrected 5\% threshold for $p$-values ( $\mathrm{Li}$ et al. 2012) $=0.002$. For categorical noise variables, $p$-values are additionally given for individual categories in comparison to the reference category (Wald test $p$-values); these are not used when computing the multiple hypothesis testing corrected 5\% threshold for $p$-values.

${ }^{e}$ Square root-transformed.

${ }^{f}$ ln-transformed.

${ }^{g}$ Squared.

${ }^{h}$ Cubic root-transformed. 


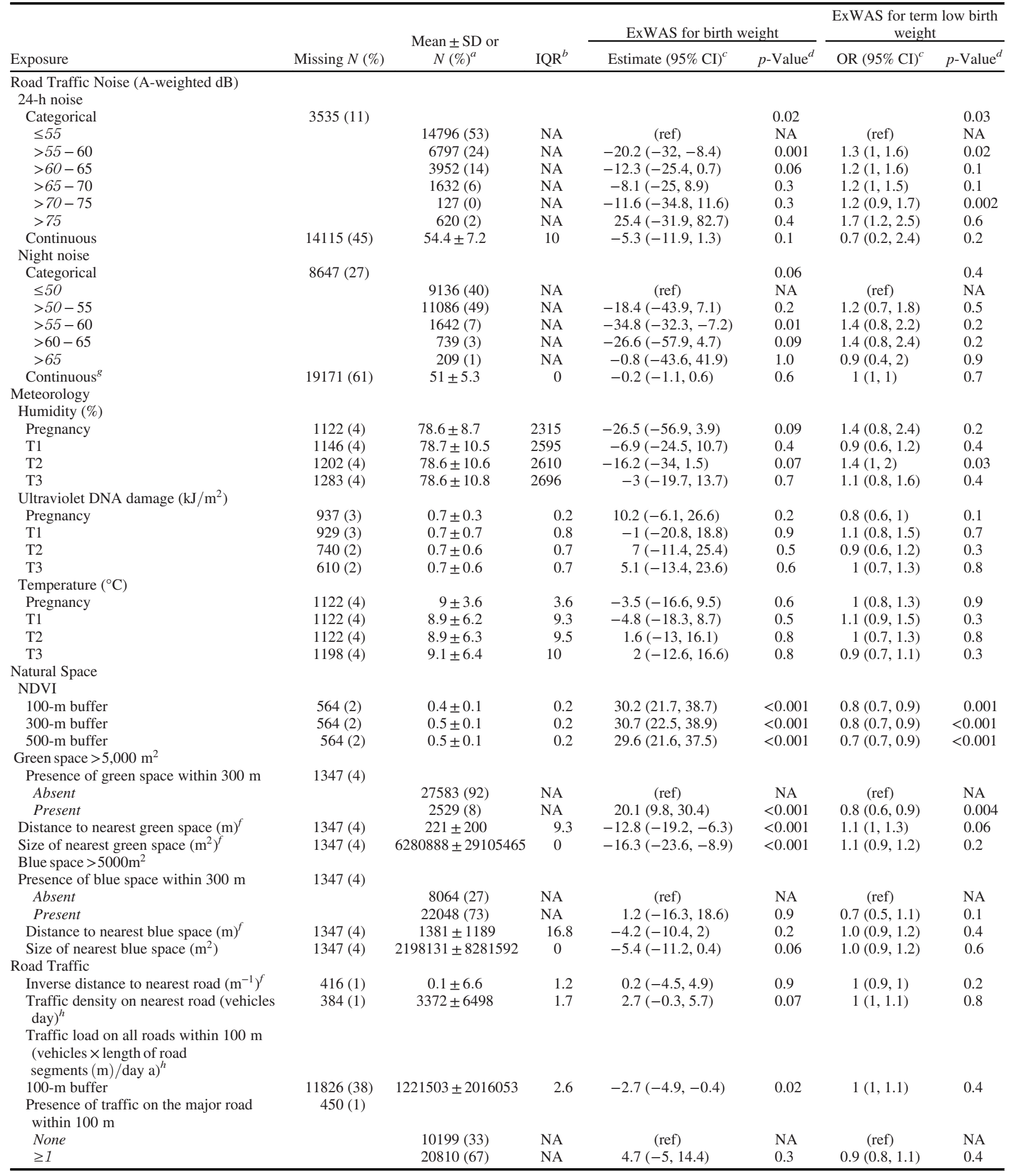

flexible version of the stepwise variable selection procedure, that, on top of testing at each step adding or removing a term to the model, also tests replacing a term by another. The method investigates a set of linear regression models and identifies an optimal model for each model possible size. The model that is finally selected is the one that minimizes the value of the root mean squared prediction error (RMSE) using 5-fold cross-validated data (i.e., the study population is randomly partitioned into five subsets; for each of these subsets, the model was trained on the other four partitions and fitted on the given left-out subset over which the 


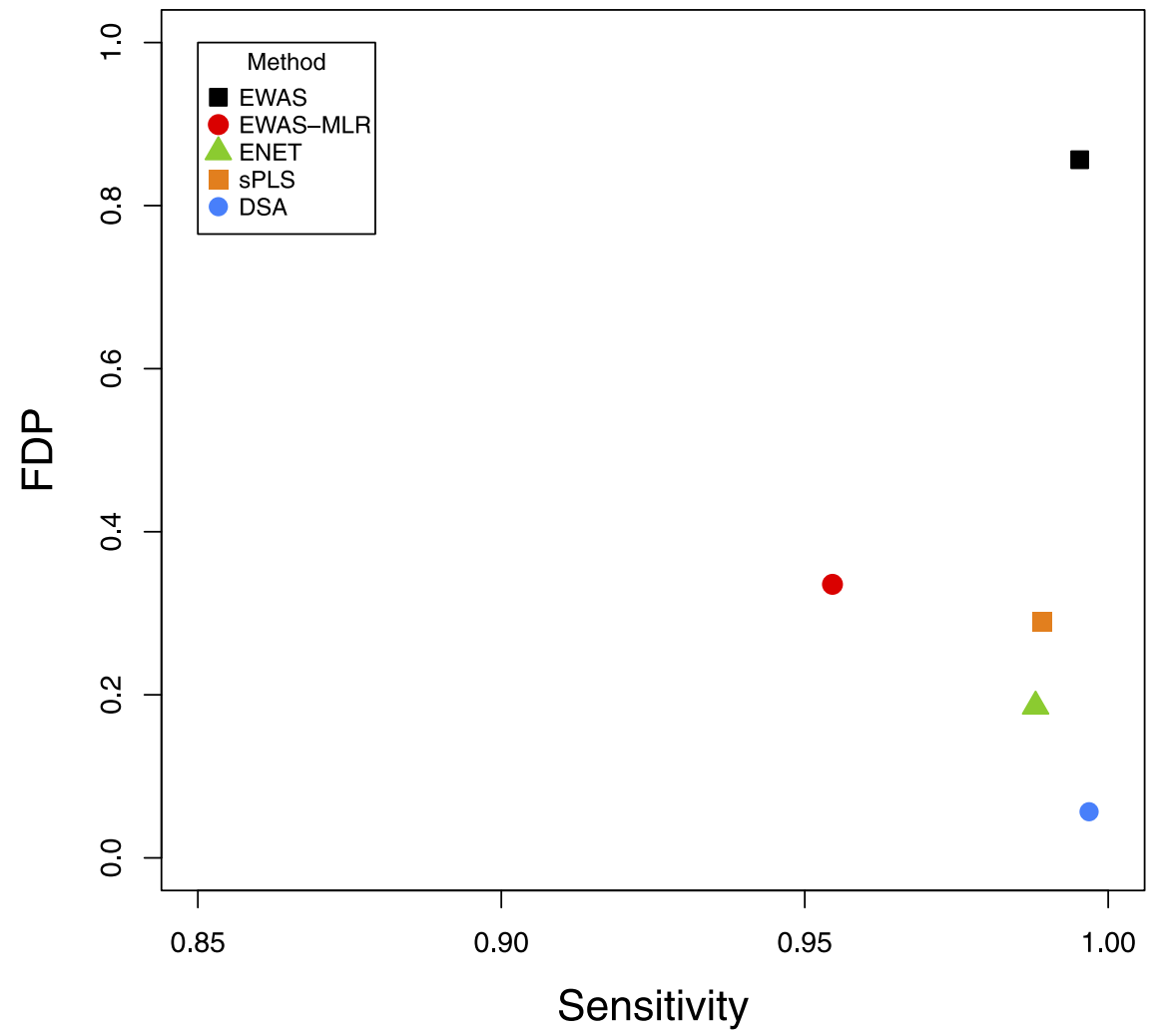

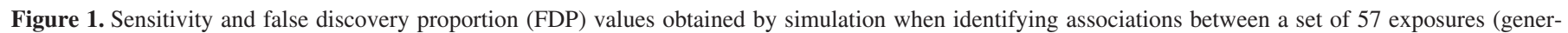

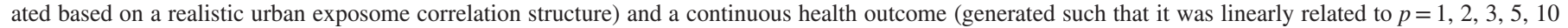

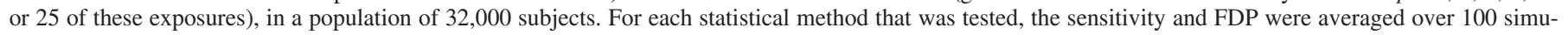
lation runs and over all six scenarios (i.e., with $p=1,2,3,5,10$ and 25 exposures influencing the outcome). More detailed results are given in Figure S1.

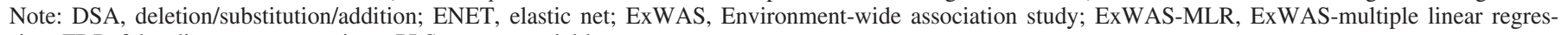
sion; FDP, false discovery proportion; sPLS, sparse partial least-squares.

RMSE was estimated). Because DSA relies on cross-validation, results can differ across calculation runs. To obtain more stable results, we adopted an ad hoc strategy. We ran each DSA model 100 times and fitted a regression model including all exposures selected in at least five runs. We removed them one by one, starting from the exposure least frequently selected in the 100 DSA runs, until the parameters of all exposures in the model were significant at a 5\% level. Our final "DSA" results correspond to the estimates of this last multiple regression model.

The DSA procedure was fitted relying on linear models for birth weight, and logistic models for TLBW. Models were adjusted for the potential confounders indicated above. As suggested in the standard procedure, we did not allow polynomial or interaction terms between exposures. Some of the urban exposome variables were redundant and displayed high correlations that were likely to hinder any multiexposures regression model, such as a given environmental indicator being averaged over several exposure windows. Hence, in DSA, we selected one exposure with high a priori plausibility per exposure indicator and ensured that no pair of exposures displayed an absolute correlation coefficient above $90 \%$ (when this happened, we selected the exposure variable with the smallest proportion of imputed values and removed the other). In total, 21 exposures were included in DSA.

In the light of systematically analyzing and reporting all associations and reducing reporting bias, we initially performed a holistic and unadjusted-for-coexposure ExWAS analysis (Table 1). By allowing to evaluate all exposures' associations with birth weight across critical periods of time, ExWAS for example may enlighten associations that were hidden in DSA because of exposures' collinearity. It also provides relevant information for future metaanalyses, which should not be restricted to associations found significant in a given study.

The ExWAS approach (Patel et al. 2010) consists of a exposure-by-exposure estimation of the association of each exposure variable with the outcome using regression models adjusted for potential confounders. Linear regression was applied for birth weight analysis, and Fisher test $p$-values were used; logistic regression was applied for TLBW analysis, and deviance test $p$-values were used. To account for multiple hypothesis testing, $p$-values were compared to $5 \%$ divided by the effective number of independent tests as defined by Li et al. (2012), which is an estimate of the number of truly independent tests that are performed, given the correlation structure of association test $p$-values (here, 25). In a second step, we included in a multivariable linear regression model all the exposure variables that were significant in ExWAS at a 5\% level after multiple hypothesis testing correction and after removing the redundant and highly correlated exposures (ExWAS-MLR) to check the stability of the exposure-health associations when accounting for confounding by coexposures (Mickey and Greenland 1989).

All analyses were performed with the $\mathrm{R}$ statistical software (version 3.4; R Development Core Team), using the rexposome package for drawing plots (https://rdrr.io/bioc/rexposome/), mice for multiple imputation, and DSA for the DSA algorithm. The simulation computations were performed using the CIMENT infrastructure (https://ciment.ujf-grenoble.fr), which is supported by the Rhône-Alpes region (GRANT CPER07_13 CIRA; http:// www.ci-ra.org). 


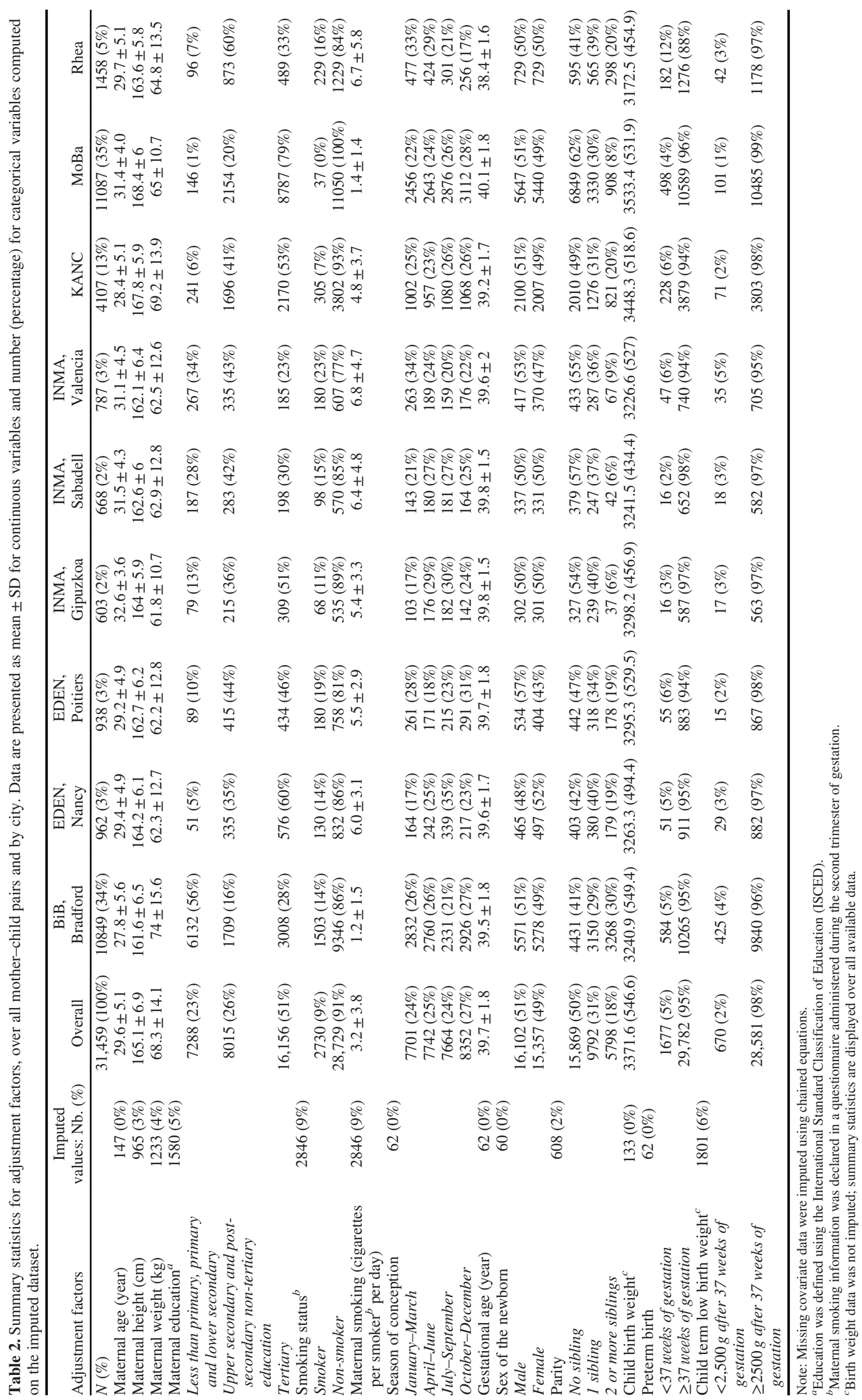



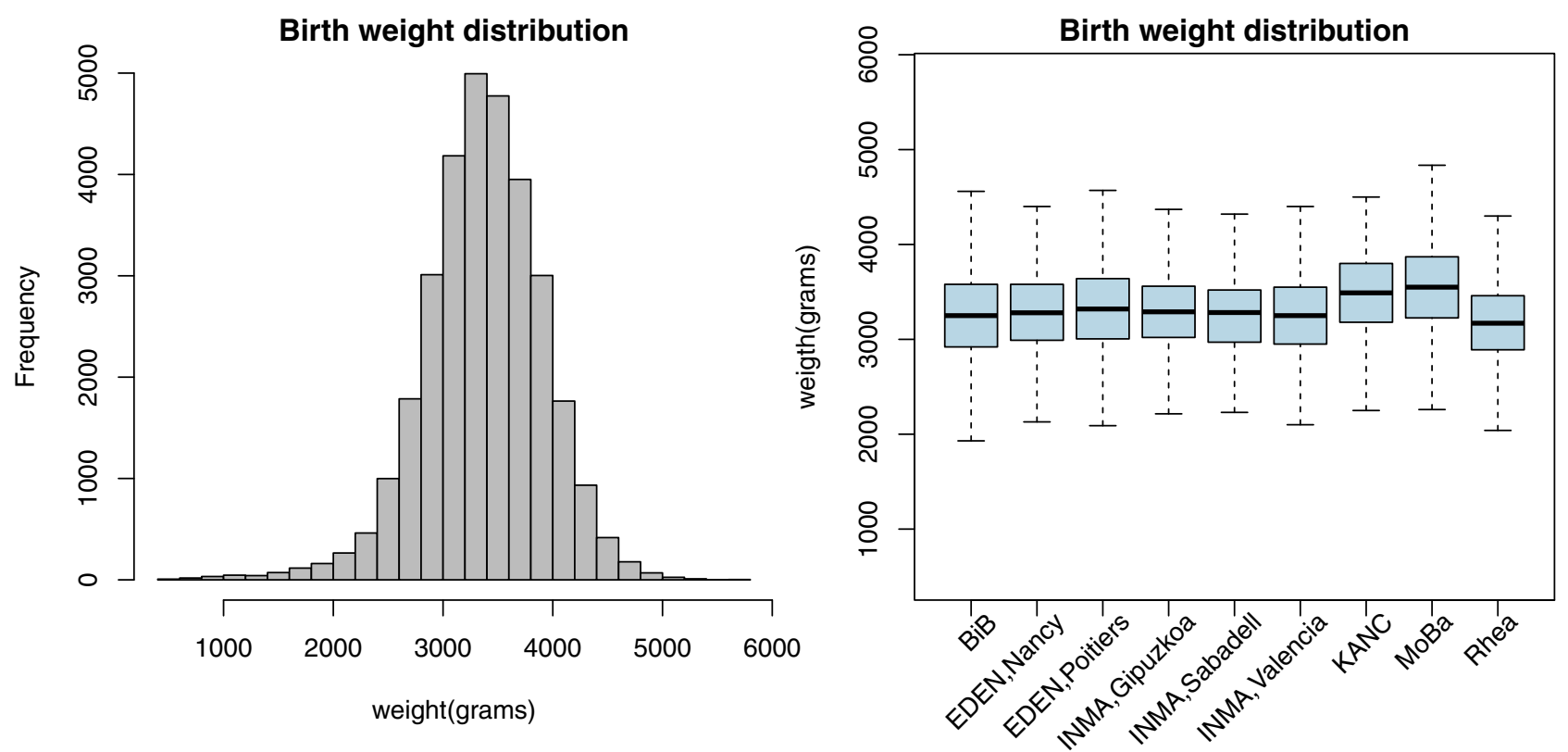

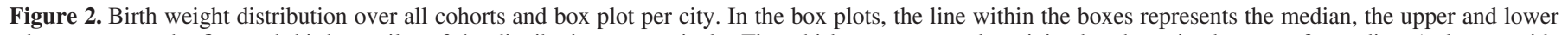

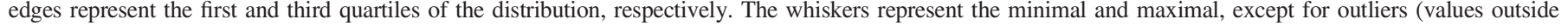
1.5 times the IQR above the upper quartile and bellow the lower quartile) that are individually displayed on the figure as dots. Note: IQR, interquartile range.

\section{Results}

\section{Study Population}

At the time of pregnancy, mothers were on average ( \pm standard deviation, SD) $29.6 \pm 5.1$ years old, with a prepregnancy weight of $68.3 \pm 14.1 \mathrm{~kg}$ and a height of $165 \pm 7 \mathrm{~cm}$ (Table 2). Most had tertiary education $(51 \%)$; $9 \%$ of women smoked during the second trimester of pregnancy (with an average of $3.2 \pm 3.8$ cigarettes smoked per day for smokers). There was a balanced distribution of pregnancies across seasons (between 24\% and $27 \%$ over the four seasons). Children were born after $39.7 \pm$ 1.8 weeks of gestation, with a 1.05 male-to-female sex ratio. These characteristics were overall similar across cities, except for maternal education and smoking.

Birth weight data was available for $31,326(96.0 \%)$ children and was on average $3,372 \pm 547$ grams, with a minimum of 465 and a maximum of 5,800 grams (Figure 2). Out of the 29,658 (94.2\%) term births, 699 (2.4\%) had a low birth weight.

\section{The Urban Exposome and Birth Weight}

In the exposure-by-exposure ExWAS analysis, 18 exposures covering 11 environmental indicators were statistically significant after $p$-value correction for multiple hypothesis testing (Table 3, Figure 3). The most significant exposures indicators were, by increasing $p$-value: NDVI [birth weight change in grams for an IQR change in exposure levels: $30.2 \mathrm{~g}$; $95 \%$ confidence interval (CI): $21.7,38.7$ for the 100 -m buffer], walkability index $(-16.7 \mathrm{~g}$; 95\% CI: $-23.2,-10.2)$, presence of public bus line (19.6 g; 95\% CI: $10.2,29.1$ for presence within $300 \mathrm{~m}$ ), facilities density $(-13.4 \mathrm{~g} ;$ 95\% CI: $-19.9,-6.9$ for the $300-\mathrm{m}$ buffer, logtransformed exposure values), building density ( $-11.9 \mathrm{~g} ; 95 \% \mathrm{CI}$ : $-17.7,-6.0$ for the $300-\mathrm{m}$ buffer, cubic root-transformed exposure values), green space (20.1 g; 95\% CI: 9.8, 30.4 for presence within $300 \mathrm{~m}), \mathrm{PM}_{2.5}$ absorbance $(-17.9 \mathrm{~g} ; 95 \% \mathrm{CI}:-27.2,-8.6$ for trimester 3 of pregnancy average, log-transformed exposure values), connectivity density $(-13.9 \mathrm{~g}$; 95\% CI: $-21.4,-6.4$ for the 300-m buffer, cubic root-transformed exposure values), land use SEI ( $-9.1 \mathrm{~g} ; 95 \% \mathrm{CI}:-14.7,-3.5$ for the $300-\mathrm{m}$ buffer),
$\mathrm{PM}_{2.5}$ (-15.0 g; 95\% CI: $-24.4,-5.6$ for the whole pregnancy average), and $\mathrm{NO}_{2}(-12.1 \mathrm{~g} ; 95 \% \mathrm{CI}:-19.7,-4.5$ for trimester 3 of pregnancy average, log-transformed exposure values). Other measures of the same environmental indicator were significant for NDVI, green space, presence of public bus line, facilities and building density, with consistent coefficients estimates.

Over the 11 exposures that were jointly included in the ExWASMLR regression model (maximal absolute pairwise correlation value: 0.71 , only NDVI, land use SEI, $\mathrm{PM}_{2.5}$ absorbance and presence of public bus line exposures remained statistically significant at a 5\% level; their coefficients were consistent with the ExWAS exposure-by-exposure models. The seven remaining exposure indicators significantly associated with birth weight did not survive adjustment by coexposure confounding, and their parameters differed substantially from those obtained in the ExWAS models (Table 3).

In the multiexposures DSA analysis, four exposures were identified for being jointly associated with birth weight (Table 4): NDVI in a 100-m buffer (birth weight change in grams for an IQR change in exposure levels: $28.0 \mathrm{~g}$; 95\% CI: 19.3, 36.6), presence of bus line within $300 \mathrm{~m}$ (14.3 g; 95\% CI: 4.7, 23.9), land use SEI in a $300-\mathrm{m}$ buffer $(-7.8 \mathrm{~g} ; 95 \% \mathrm{CI}:-13.4,-2.2)$ and traffic density on the nearest road from pregnancy home address (birth weight change in grams for an IQR change in cubic roottransformed exposure levels: $3.7 \mathrm{~g}$; 95\% CI: 0.6, 6.7).

\section{The Urban Exposome and TLBW}

In ExWAS, the exposure indicators associated with TLBW risk (Table 5, Figure 4) were, by increasing $p$-value, building density [odds ratio $(\mathrm{OR})=1.2$; 95\% CI: $1.1,1.4$ for the $300-\mathrm{m}$ buffer, cubic root-transformed exposure values] and NDVI $(\mathrm{OR}=0.8$; 95\% CI: $0.7,0.9$ for the $100-\mathrm{m}$ buffer). For both environmental indicators, averaged measures over other buffers were statistically significant, with consistent OR estimates. No exposure was significant at a 5\% level in the ExWAS-MLR model that aimed to adjust for potential confounding by coexposures.

In the DSA analysis, the only exposure that was identified for being associated with TLBW was NDVI in a 100-m buffer, with 
Table 3. Results of ExWAS (i.e., exposure by exposure linear regression models) and ExWAS-MLR (i.e., multiple linear regression model simultaneously including all exposures selected in ExWAS after excluding redundant variables) testing the association between the urban exposome and continuous birth weight, adjusted for potential confounders. Only parameters associated with exposures with a multiple hypothesis testing-corrected $p$-value in ExWAS below $5 \%$ are reported.

\begin{tabular}{|c|c|c|c|c|c|c|}
\hline \multirow[b]{2}{*}{ Exposure } & \multirow[b]{2}{*}{ Unit } & \multirow[b]{2}{*}{$\mathrm{IQR}^{a}$} & \multicolumn{2}{|l|}{ ExWAS } & \multicolumn{2}{|c|}{ ExWAS-MLR } \\
\hline & & & Estimate $(95 \% \mathrm{CI})^{b}$ & $p$-Value ${ }^{c}$ & Estimate $(95 \% \mathrm{CI})^{b}$ & $p$-Value \\
\hline \multicolumn{7}{|l|}{ Built Environment } \\
\hline Building density in a $100-\mathrm{m}$ buffer $^{d}$ & $\mathrm{~m}^{2}$ built $/ \mathrm{km}^{2}$ & 144.9 & $-12.3(-18.4,-6.3)$ & $<0.001$ & $\mathrm{NA}^{e}$ & \\
\hline Building density in a $300-\mathrm{m}$ buffer $^{d}$ & $\mathrm{~m}^{2}$ built $/ \mathrm{km}^{2}$ & 138.7 & $-11.9(-17.7,-6.0)$ & $<0.001$ & $6.3(-3.1,15.6)$ & 0.2 \\
\hline Connectivity density in a $300-\mathrm{m}$ buffer $^{d}$ & Intersections $/ \mathrm{km}^{2}$ & 6.179 & $-13.9(-21.4,-6.4)$ & $<0.001$ & $0.8(-9.8,11.5)$ & 0.9 \\
\hline Land use Shannon's Evenness Index & Index & 0.177 & $-9.1(-14.7,-3.5)$ & 0.001 & $-8.6(-16.2,-1.1)$ & 0.02 \\
\hline Presence of public bus line within $300 \mathrm{~m}$ & Binary & NA & $19.6(10.2,29.1)$ & $<0.001$ & $13.6(3.6,23.5)$ & 0.007 \\
\hline Presence of public bus line within $500 \mathrm{~m}$ & Binary & NA & $27.4(17.4,37.3)$ & $<0.001$ & $\mathrm{NA}^{e}$ & \\
\hline Facilities density ${ }^{f}$ & Facilities $/ \mathrm{km}^{2}$ & 5.094 & $-13.4(-19.9,-6.9)$ & $<0.001$ & $2.2(-8.5,13.0)$ & 0.7 \\
\hline Facility richness & Facility types $/ \mathrm{km}^{2}$ & 0.187 & $-13.5(-19.6,-7.3)$ & $<0.001$ & $\mathrm{NA}^{e}$ & \\
\hline Walkability index & Index & 0.368 & $-16.7(-23.2,-10.2)$ & $<0.001$ & $0.5(-13.4,14.3)$ & 0.9 \\
\hline \multicolumn{7}{|l|}{ Air pollution } \\
\hline $\mathrm{NO}_{2}$ in trimester 3 of pregnancy ${ }^{f}$ & $\mu \mathrm{g} / \mathrm{m}^{3}$ & 0.543 & $-12.1(-19.7,-4.5)$ & 0.002 & $4.8(-5.3,14.8)$ & 0.3 \\
\hline $\mathrm{PM}_{2.5}$ absorbance in trimester 3 of pregnancy ${ }^{f}$ & $10^{-5} / \mathrm{m}$ & 0.551 & $-17.9(-27.2,-8.6)$ & $<0.001$ & $-12.1(-23.8,-0.5)$ & 0.04 \\
\hline $\mathrm{PM}_{2.5}$ pregnancy average & $\mu \mathrm{g} / \mathrm{m}^{3}$ & 0.339 & $-15.0(-24.4,-5.6)$ & 0.002 & $-2.6(-13.1,7.9)$ & 0.6 \\
\hline \multicolumn{7}{|l|}{ Natural Space } \\
\hline NDVI in a $100-m$ buffer & Index & 0.203 & $30.2(21.7,38.7)$ & $<0.001$ & $32.1(18.7,45.6)$ & $<0.001$ \\
\hline NDVI in a $300-\mathrm{m}$ buffer & Index & 0.175 & $30.7(22.5,38.9)$ & $<0.001$ & $\mathrm{NA}^{e}$ & \\
\hline NDVI in a $500-\mathrm{m}$ buffer & Index & 0.162 & $29.6(21.6,37.5)$ & $<0.001$ & $\mathrm{NA}^{e}$ & \\
\hline Presence of green space within 300-m & Binary & NA & $20.1(9.8,30.4)$ & $<0.001$ & $10.3(-1.1,21.7)$ & 0.07 \\
\hline Distance to nearest green space ${ }^{f}$ & $\mathrm{~m}$ & 9.319 & $-12.8(-19.2,-6.3)$ & $<0.001$ & $\mathrm{NA}^{e}$ & \\
\hline Size of nearest green space $f$ & $\mathrm{~m}^{2}$ & 0.006 & $-16.3(-23.6,-8.9)$ & $<0.001$ & $\mathrm{NA}^{e}$ & \\
\hline
\end{tabular}

Note: All buffer areas and distances are relative to the pregnancy residence unless otherwise indicated. Missing exposure and covariate data were imputed using chained equations. CI, confidence interval of the coefficient estimate; ExWAS, exposome-wide association study; ExWAS-MLR, multiple linear regression model simultaneously including all exposures selected in ExWAS after excluding redundant variables; IQR, interquartile range; NA, not applicable; NDVI, normalized difference vegetation index; $\mathrm{NO}_{2}$, nitrogen dioxide; $\mathrm{PM}_{2.5}$, particulate matter in the (ambient) air with an aerodynamical diameter below 2.5 micrometers.

${ }^{a}$ The IQR is computed over continuous exposures only, after imputing missing values and transforming data to approach normality.

${ }^{b}$ Difference in mean birth weight in association with an IQR increase in (imputed and transformed to approach normality) exposure (continuous variables), or relative to the reference category (binary and categorical variables), adjusted for a city effect, gestational age (simple and quadratic terms), sex of the newborn, parity, maternal height and weight before pregnancy, maternal smoking, maternal education, and season of conception.

${ }^{c}$ Uncorrected $p$-values testing the global significance of the variable, obtained using a Wald test (continuous or binary variables) or a likelihood ratio test (categorical noise variables). Multiple hypothesis testing corrected 5\% threshold for $p$-values (Li et al. 2012) $=0.002$.

${ }^{d}$ Square root-transformed.

${ }^{e}$ The exposure was removed from the multivariate ExWAS-MLR model for it was redundant with another exposure selected in ExWAS, i.e. either the same environmental indicator was measured over several exposure windows or with various methods; or it displayed an absolute correlation $>0.9$ with another exposure. NDVI in a 100 -m buffer was selected to the detriment of NDVI in a 300-m or 500-m buffer; the presence of green space within $300 \mathrm{~m}$ was selected to the detriment of size and distance to nearest green space; the presence of public bus line within $300 \mathrm{~m}$ was selected to the detriment of presence of public bus line within $500 \mathrm{~m}$; building density in a 300 -m buffer was selected to the detriment of building density in a 100-m buffer; and facilities densitywas selected to the detriment of facility richness.

fln-transformed.

an OR of low birth weight associated with an IQR change in exposure of 0.9 (95\% CI: 0.7, 0.9).

\section{Discussion}

To the best of our knowledge, we have conducted here the largest analysis of the association between the urban exposome and birth weight. We accounted for the multiplicity of exposures that were tested, and we reported statistically significant exposure associations with fetal growth, as well as nonsignificant associations to be used for future meta-analyses. The most statistically significant and consistent associations were for green space estimated as NDVI with birth weight and TLBW (with an increased fetal growth with increasing NDVI exposure). When we accounted for potential confounding by coexposures, NDVI, presence of public bus line, and traffic density displayed a significant positive association with birth weight, whereas land use SEI and $\mathrm{PM}_{2.5}$ absorbance displayed a significant negative association with birth weight. Furthermore, we observed statistically significant (unadjusted for coexposures) positive associations with birth weight for presence of green space; negative associations with birth weight for walkability index, facilities density, building density, connectivity density, $\mathrm{PM}_{2.5}$ and $\mathrm{NO}_{2}$, some of which may warrant further investigation.

Our results on NDVI and fetal growth are consistent with previous studies that showed an increase in birth weight with increasing levels of NDVI (Cusack et al. 2017; Dzhambov et al. 2014; Hystad et al. 2014). Green space has been associated with many beneficial health effects, including reduced premature mortality, better mental health, and better cognitive functioning (Nieuwenhuijsen et al. 2017).

We found that increasing levels of $\mathrm{PM}_{2.5}$ were associated with a decreased birth weight; however, we could not replicate the associations of this air pollutant with TLBW that were reported before in a larger study in Europe overlapping this study population (Pedersen et al. 2013). The level of evidence regarding negative effects of air pollution on birthweight is strong, and a number of meta-analyses have reported negative effects of air pollution, particularly PM, on birth weight (Lamichhane et al. 2015; Stieb et al. 2012). The DSA analysis did not identify $\mathrm{PM}_{2.5}$, but traffic density was identified, which could be viewed as a proxy of the same exposure to road pollution; however, the association was in the opposite direction to what was expected. We do not have an explanation for why this association occurred. As in various other studies, we did not observe an association between road traffic noise and (low) birth weight (Nieuwenhuijsen et al. 2017). Although a few studies found an association between temperature and (term) low birth weight, we did not, which most likely reflects the inconsistent nature of the relationship, the use of different exposure periods (days vs. months), and the focus of previous studies on extreme temperatures, whereas we did not. Furthermore, we did not consider nonlinear relationships. 


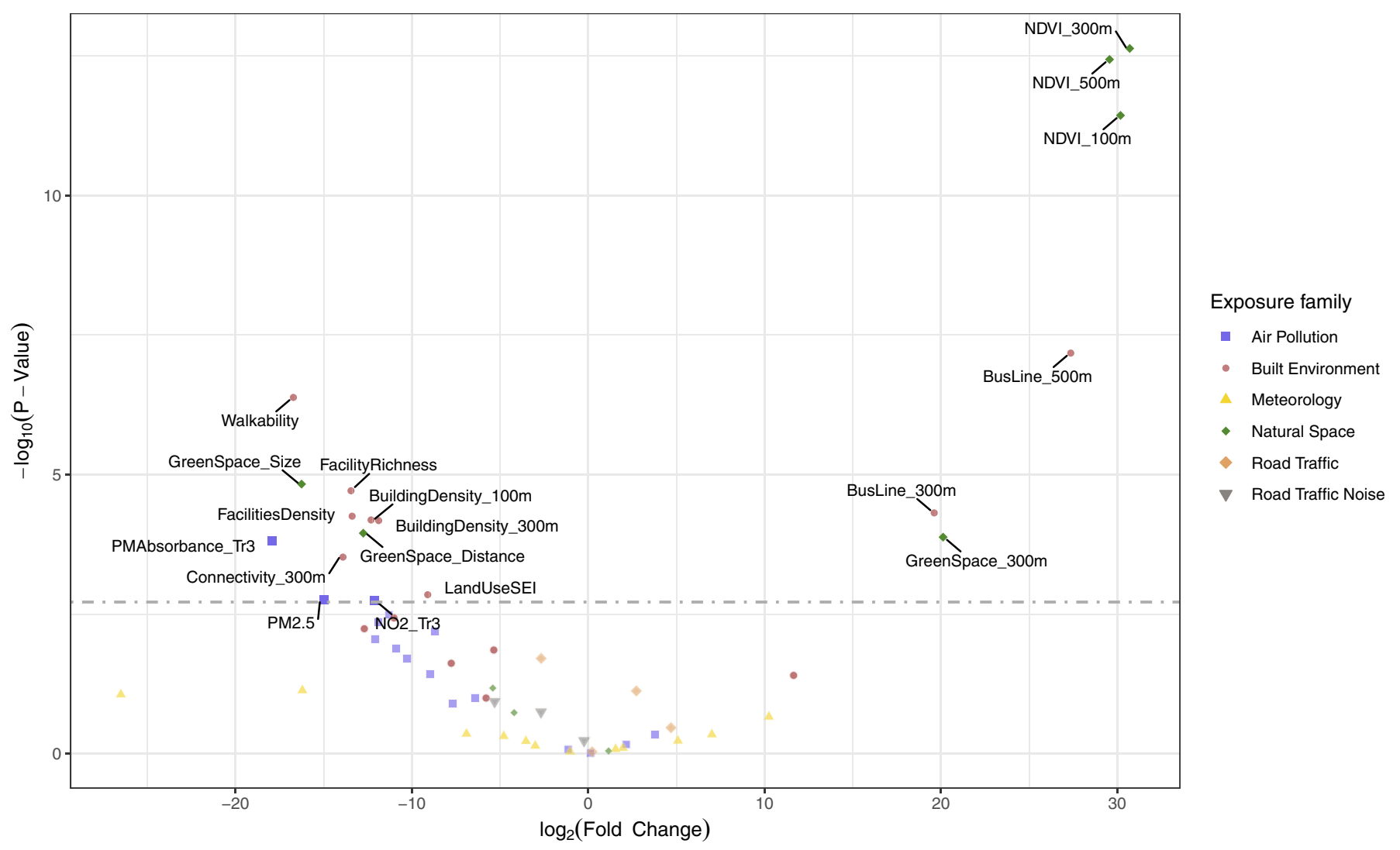

Figure 3. Volcano plot giving the fold change coefficient estimate vs. the $p$-value obtained with the ExWAS analysis of the association of all exposures of the urban exposome with birth weight. The grey dotted-dashed line represents the multiple hypothesis testing corrected 5\% threshold obtained with the Li et al. method (Li et al. 2012). Exposures with a $p$-value lower than this threshold have their names on the graph (see Table 3 for more results). Other exposures are displayed in faded colors; see Table 2 for all ExWAS results. Note: All buffer areas and distances are relative to the pregnancy residence unless otherwise indicated. Missing exposure and covariate data were imputed using chained equations. BuildingDensity_100 m, building density in a 100-m buffer; BuildingDensity_300 m, building density in a $300-\mathrm{m}$ buffer; BusLine_300 m, presence of bus line within 300-m; BusLine_500 m, presence of bus line within 500-m; Connectivity_300 m, connectivity density in a 300-m buffer; FacilitiesDensity, facilities density in a 300-m buffer; FacilityRichness, facility richness in a 300-m buffer; GreenSpace_Distance, distance to nearest green space; GreenSpace_Size, size of nearest green space; GreenSpace_300 m, presence of green space within $300 \mathrm{~m}$; LandUseSEI, land use Shannon's Evenness Index in a 300-m buffer; NDVI_100 m, normalized difference vegetation index in a 100-m buffer; NDVI_300 m, normalized difference vegetation index in a 300-m buffer; NDVI_500 m, normalized difference vegetation index in a 500-m buffer; $\mathrm{NO}_{2}$ Tr3, nitrogen dioxide averaged over the third trimester of pregnancy; $\mathrm{PM}_{2.5}$, particulate in the (ambient) air 2.5 micrometers or less in aerodynamical diameter averaged over the whole pregnancy; PMAbsorbance_Tr3, particulate in the (ambient) air 2.5 micrometers or less in aerodynamical diameter absorbance averaged over the third trimester of pregnancy; Walkability, walkability index in a 300-m buffer.

Associations between built environment exposures such as building density, walkability index, presence of public bus line, facilities density, connectivity density, and land use SEI with birth weight are novel, as very few studies have been conducted on the topic (Gascon et al. 2016). The presence of bus line association with birth weight needs to be interpreted with caution, given that $45 \%$ of the data was imputed (whereas for the other hits, no more than $15 \%$ of the data was imputed).

Table 4. Results of DSA testing the association of the urban exposome and continuous birth weight, adjusted for potential confounders.

\begin{tabular}{llcr}
\hline Exposure & Unit & IQR $^{a}$ & ${\text { Estimate }(95 \% \text { CI })^{b}}^{p}$ \\
\hline $\begin{array}{l}\text { Built Environment } \\
\text { Land use Shannon's Evenness Index }\end{array}$ & Index & 0.177 & $-7.8(-13.4,-2.2)$ \\
$\quad \begin{array}{l}\text { Presence of public bus line within 300 m } \\
\text { Natural Space }\end{array}$ & Binary & NA & $14.3(4.7,23.9)$ \\
$\quad$ NDVI in a 100-m buffer & Index & 0.203 & 0.006 \\
$\begin{array}{l}\text { Road Traffic } \\
\text { Traffic density in the nearest road }\end{array}$ & Vehicles/day & 1.652 & $28.0(19.3,36.6)$ \\
\hline
\end{tabular}

Note: All buffer areas and distances are relative to the pregnancy residence unless otherwise indicated. Missing exposure and covariate data were imputed using chained equations. CI, confidence interval of the coefficient estimate; DSA, deletion/substitution/addition algorithm; IQR, interquartile range; NA, not applicable; NDVI, normalized difference vegetation index.

${ }^{a}$ The IQR is computed over continuous exposures only, after imputing missing values and transforming data to approach normality.

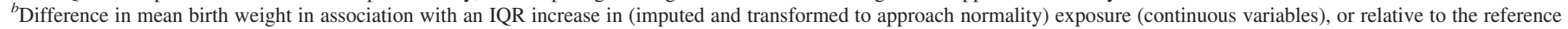

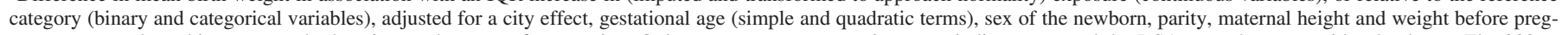

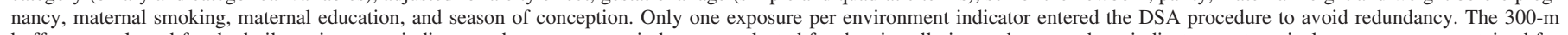
buffer was selected for the built environment indicators; the pregnancy window was selected for the air pollution and meteorology indicators; categorical exposures were retained for

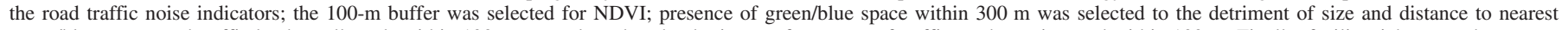

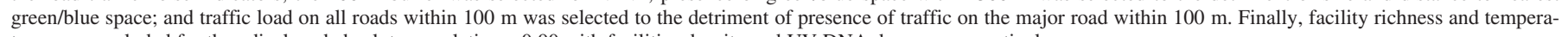
ture were excluded for they displayed absolute correlation $>0.90$ with facilities density and UV DNA damage, respectively.

${ }^{c}$ Cubic root-transformed. 
Table 5. Results of ExWAS (i.e., exposure by exposure logistic regression models) and ExWAS-MLR (i.e., multiple logistic regression model simultaneously including all exposures selected in ExWAS after excluding redundant variables) testing the association between the urban exposome and term low birth weight, adjusted for potential confounders. Only results associated with exposures with a multiple hypothesis testing-corrected $p$-value in ExWAS below 5\% are reported.

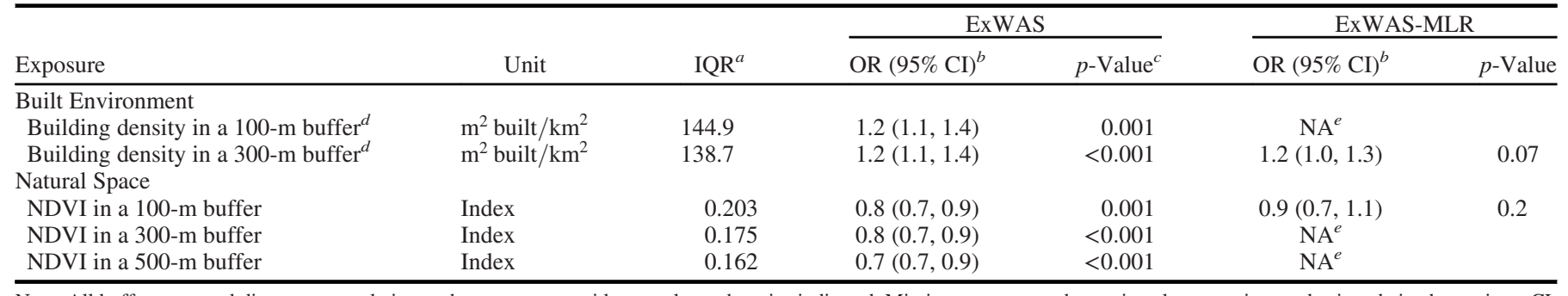

Note: All buffer areas and distances are relative to the pregnancy residence unless otherwise indicated. Missing exposure and covariate data were imputed using chained equations. CI, confidence interval of the coefficient estimate; ExWAS, exposome-wide association study; ExWAS-MLR, multiple linear regression model simultaneously including all exposures selected in ExWAS after excluding redundant variables; IQR, interquartile range; NA, not applicable; NDVI, normalized difference vegetation index; OR, odds ratio.

${ }^{a}$ The IQR is computed over continuous exposures only, after imputing missing values and transforming data to approach normality.

${ }^{b}$ Odds ratio (OR) for term low birth weight in association with an IQR increase in (imputed and transformed to approach normality) exposure (continuous variables), or relative to the reference category (binary and categorical variables), adjusted for a city effect, gestational age (simple and quadratic terms), sex of the newborn, parity, maternal height and weight before pregnancy, maternal smoking, maternal education, and season of conception.

${ }^{c}$ Uncorrected $p$-values testing the global significance of the variable, obtained using a Wald test (continuous or binary variables) or a deviance test (categorical noise variables). Multiple hypothesis testing corrected 5\% threshold for $p$-values (Li et al. 2012) $=0.002$.

${ }^{d}$ Square root-transformed.

${ }^{e}$ The exposure was removed from the multivariate ExWAS-MLR model for it was redundant with another variable selected in ExWAS, i.e. either the same environmental indicator was measured over several exposure windows or with various methods; or it displayed an absolute correlation $>0.9$ with another exposure. NDVI in a 100 -m buffer was selected to the detriment of NDVI in a 300-m or a 500-m buffer; building density in a 300-m buffer was selected to the detriment of building density in a 100 -m buffer.

Only one study so far examined the association of a built environment measure of walkability and birth weight and also found that an increase in walkability score was associated with a decreased birth weight (Hystad et al. 2014). In our study, the associations with built environment exposures were not consistent across our statistical analyses, and most effects were not statistically significant in our analyses after adjusting for other exposures.

Only a couple of studies so far have examined the relationship between multiple environmental exposures and birth weight.

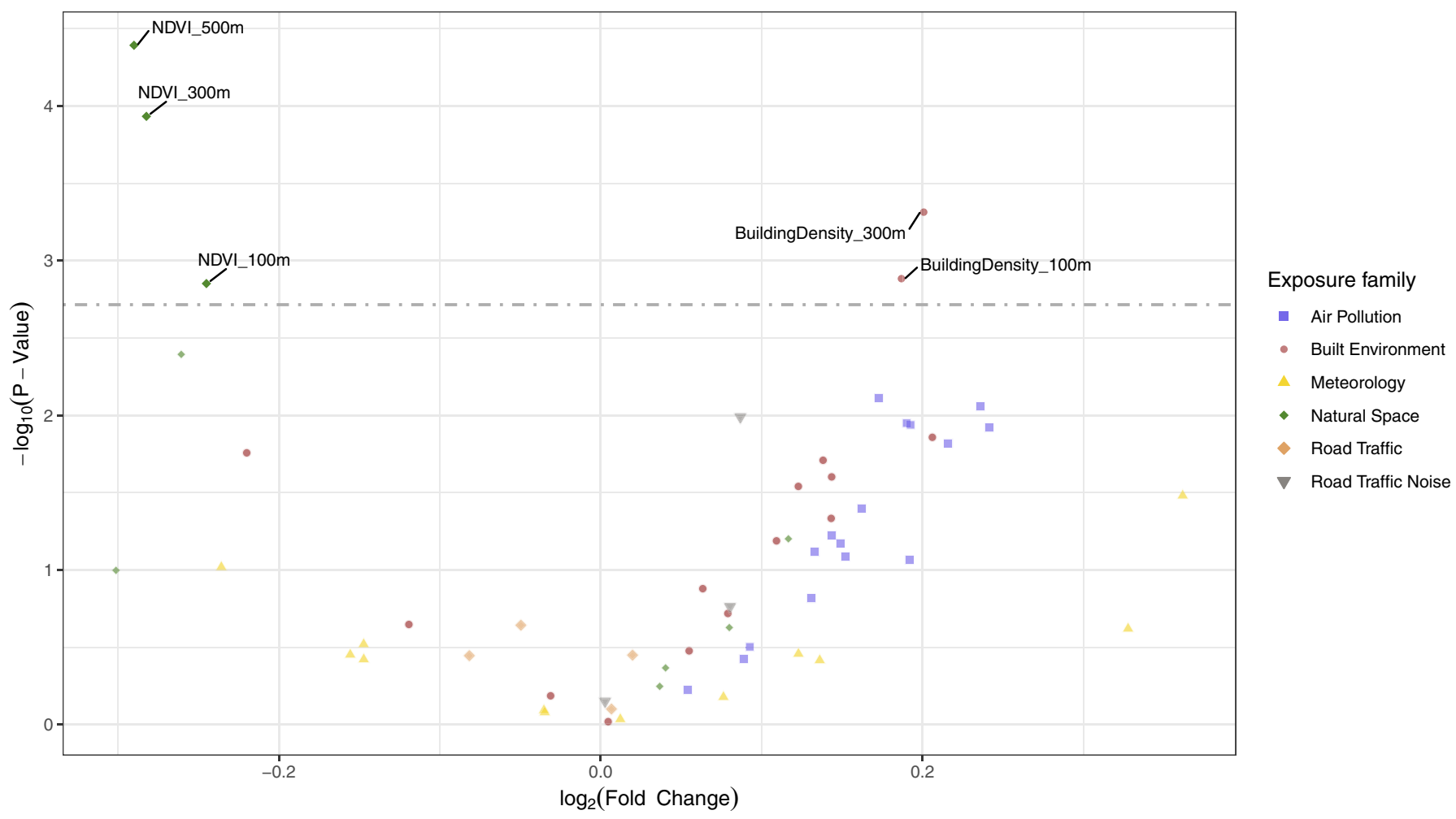

Figure 4. Volcano plot giving the fold change coefficient estimate vs. the $p$-value obtained with the ExWAS analysis of the association of all exposures of the urban exposome with term low birth weight. The grey dotted-dashed line represents the multiple hypothesis testing corrected 5\% threshold obtained with the Li et al. method ( $\mathrm{Li}$ et al. 2012). Exposures with a $p$-value lower than this threshold have their names on the graph (see Table 5 for more results). Other exposures are displayed in faded colors; see Table 2 for all ExWAS results. Note: All buffer areas and distances are relative to the pregnancy residence unless otherwise indicated. Missing exposure and covariate data were imputed using chained equations. BuildingDensity_100 m, building density in a $100-\mathrm{m}$ buffer; BuildingDensity_300 m, building density in a 300-m buffer; NDVI_100 m, normalized difference vegetation index in a 100-m buffer; NDVI_300 m, normalized difference vegetation index in a 300-m buffer; NDVI_500 m, normalized difference vegetation index in a 500-m buffer. 
Hystad et al. (2014) examined the relationship between green space, noise, air pollution and walkability. They found that in general, in their fully adjusted models, the association between air pollution, neighborhood walkability, and park distance with birth weight largely disappeared with the inclusion of greenness exposures, as we found in our analyses, and the effects of noise exposures were reduced by approximately $50 \%$ (in our study, no association was found even when not adjusting for greenness). Birth weight increased with increasing NDVI, as in our analyses. Dadvand et al. examined simultaneously the effects of distance to roads, air pollution, heat, noise, and green space on TLBW and found that air pollution $\left(\mathrm{PM}_{2.5}\right)$ and heat exposures increased the risk for TLBW and together explained about one-third of the association between residential proximity to major roads and TLBW (Dadvand et al. 2014).

The urban environment is complex, with multiple exposures and determinants of exposure (Nieuwenhuijsen 2016) that are not always easy to disentangle in these types of analyses (Agier et al. 2016). Further in-depth hierarchical or analyses of other types than regression may provide a better insight in the underlying determinants and pathways, but these analyses fall outside the scope of this paper.

Our main goal was to identify exposures that have an effect on our outcome of interest and to estimate their effects in an exposome context when considering simultaneously a large number of exposures. Confounding by coexposures is one of the main challenges in such settings, especially when exposures are highly correlated. In addition, a covariate can be a confounder for one exposure-outcome relation but a mediator or collider for another. We chose to rely on agnostic methods for variable selection in order to cope with the fact that not all exposures can be included in the same model, and no specific assumption on coexposure confounders could be made for all the exposures of the urban exposome.

As part of the study, we examined in a simulation study the expected performance of several variable selection methods in an exposome context and selected DSA because it provided the best performance in the sense of identifying the truly associated exposures. Although in smaller-size exposome studies (e.g., in 1,200 subjects), false discoveries is an issue that no statistical method can easily overcome (Agier et al. 2016), in larger studies such as this one where we considered 32,000 mother-child pairs and a smaller number of covariates than in Agier et al., the risk of false-positive findings was expected to be low with DSA (5\% in the simulation analyses). The efficiency of a method in real settings is usually lower, typically because of a lower explanatory power of exposures and because of the exposure-outcome relationship that may not be linear. Power may be even lower when considering binary outcomes with low prevalence such as TLBW. Yet, given the performance of the DSA approach in our simulation, we have a good confidence in its higher performance in comparison with ExWAS. ExWAS was used to allow reporting of associations using an approach comparable to former exposure-by-exposure studies and thus enable future metaanalysis.

The strengths of the study include the design, the availability of many important adjustment factors and of many individuallevel exposures, the detailed and standardized exposure assessment, and the robust statistical methods applied after investigating their efficiency in a typical urban exposome context. In addition, we reported results for all exposures from the ExWAS exposure-birth weight and TLBW association analyses, such that they can be used in future meta-analyses and fully contribute to the weight of the evidence for any exposure investigated here. Several weaknesses need to be mentioned, such as not investigating statistical interactions or nonlinear association of exposures, not taking into account daily mobility, any mediation effects, and not accounting for measurement error in the exposures. Despite the wide range of exposures investigated in this study, the list is not exhaustive, and we may have missed some important urban environmental factors or adjustment factors for fetal growth.

Our analyses provided further insight into the challenges faced by exposome research, including those related to statistical analyses and interpretation of the results. The work has confirmed some of the previous associations that have been reported on the positive effects of green space on birth weight but could not confirm some of the previous findings on air pollution and birth weight, potentially highlighting a confounding effect of green space on the air pollution association with birth weight. Our results on road traffic noise and temperature add to the inconsistent evidence on the topic, and the novel aspects of this study are possible associations between built environment exposures and birth weight, such as land use SEI and presence of public bus line.

\section{Acknowledgments}

The research leading to these results has received funding from the European Community's Seventh Framework Programme (FP7/ 2007-2013) under Grant Agreement No. 308333 - the HELIX project. We thank M. Cirach for her advice on the air pollution data, the ESCAPE study for providing regression models to estimate air pollution levels, the PHENOTYPE study for proving the methodology to estimate green space exposure, and the TAPAS project for providing the methodology to estimate the built environment measures.

We are grateful to all the participating families in the six countries who took part in this cohort study (BiB, EDEN, INMA, KANC, MoBa, and Rhea cohorts), but especially those families who came in for a clinical examination of their children, who also donated blood and urine to this study. We are equally grateful to all the fieldworkers for their dedication and efficiency. A full roster of the INMA and Rhea project investigators can be found online.

Regarding the EDEN cohort, we thank S. Brishoual, A. Brunet, and M. Grosdenier (Poitiers Biobank, CRB BB-0033-00068, Poitiers, France) for biological sample management and F. Millot (principal investigator), P-J. Saulnier, E. Migault, M. Grellier Boue, and S. Bertin (Clinical Investigation Center, Inserm CIC1402, CHU de Poitiers, Poitiers, France) for planification and investigational actions. We are also grateful to V. FerrandRigallaud and N. Gorry (CHU de Poitiers, Poitiers, France) for administrative assistance. $\mathrm{BiB}$ is only possible because of the enthusiasm and commitment of the children and parents in BiB. We are grateful to all the participants, health professionals, and researchers who have made $\mathrm{BiB}$ happen. We thank E.P. Uphoff for her role in data collection. The study has received funding from the European Community's Seventh Framework Programme (FP7/ 2007-2013) under Grant Agreement No. 308333-the HELIX project-for data collection and analyses. The HELIX program built on six existing cohorts that received previous funding, including the major cohorts listed here. INMA data collections were supported by grants from the Instituto de Salud Carlos III, CIBERESP, the Conselleria de Sanitat, Generalitat Valenciana, Department of Health of the Basque Government; the Provincial Government of Gipuzkoa, and the Generalitat de CatalunyaCIRIT. KANC was funded by the grant of the Lithuanian Agency for Science Innovation and Technology (6-04-2014_31V-66). $\mathrm{MoBa}$ (Norwegian Mother and Child Cohort Study) is supported by the Norwegian Ministry of Health and the Ministry of Education and Research, NIH/NIEHS (Contract No. N01-ES- 
75558), and the U.S. National Institutes of Health (NIH) National Institute of Environmental Health Sciences (NIEHS; Contract No. N01-ES-75558), and National Institute of Neurological Disorders and Stroke (Grant No. 1 UO1 NS 047537-01 and Grant No. 2 UO1 NS 047537-06A1). The Rhea project was financially supported by European projects (EU FP6-2003-Food-3-NewGeneris, EU FP6.STREP Hiwate, EU FP7 ENV.2007·1.2.2.2, Project No. 211250 Escape, EU FP7-2008-ENV-1·2.1·4 Envirogenomarkers, EU FP7-HEALTH-2009-single stage CHICOS, EU FP7 ENV. 2008.1.2.1.6, Proposal No. 226285 ENRIECO, EUFP7-HEALTH2012 Proposal No. 308333 HELIX, FP7 European Union Project No. 264357 MeDALL), and the Greek Ministry of Health (Program of Prevention of Obesity and Neurodevelopmental Disorders in Preschool Children, in Heraklion district, Crete, Greece: 2011-2014; "Rhea Plus": Primary Prevention Program of Environmental Risk Factors for Reproductive Health, and Child Health: 2012-15). L.C. received additional funding from the Southern California Environmental Health Sciences Center (Grant No. P30ES007048) funded by NIEHS. We acknowledge the support of the program for international scientific collaborations of Région Rhône-Alpes-Auvergne.

P.D. is funded by a Ramón y Cajal fellowship (RYC-201210995) awarded by the Spanish Ministry of Economy and Finance.

ISGlobal is a member of the Agency for the Research Centres of Catalonia (CERCA) Programme, Generalitat de Catalunya.

\section{References}

Agier L, Portengen L, Chadeau-Hyam M, Basagaña X, Giorgis-Allemand L, Siroux $V$, et al. 2016. A systematic comparison of linear regression-based statistical methods to assess exposome-health associations. Environ Health Perspect 124(12):1848-1856, PMID: 27219331, https://doi.org/10.1289/EHP172.

Beelen R, Hoek G, Vienneau D, Eeftens M, Dimakopoulou K, Pedeli X, et al. 2013. Development of $\mathrm{NO}_{2}$ and $\mathrm{NO} x$ land use regression models for estimating air pollution exposure in 36 study areas in Europe - The ESCAPE project. Atmos Environ 72:10-23, https://doi.org/10.1016/j.atmosenv.2013.02.037.

Chatzi L, Leventakou V, Vafeiadi M, Koutra K, Roumeliotaki T, Chalkiadaki G, et al. 2017. Cohort profile: The Mother-Child Cohort in Crete, Greece (Rhea Study). Int J Epidemiol 46(5):1392-1393k, PMID: 29040580, https://doi.org/10.1093/ije/dyx084.

Cusack L, Larkin A, Carozza S, Hystad P. 2017. Associations between residential greenness and birth outcomes across Texas. Environ Res 152:88-95, PMID: 27743971, https://doi.org/10.1016/j.envres.2016.10.003.

Dadvand P, Ostro B, Figueras F, Foraster M, Basagaña X, Valentín A, et al. 2014. Residential proximity to major roads and term low birth weight. Epidemiology 25(4):518-525, PMID: 24787556, https://doi.org/10.1097/EDE.0000000000000107.

Dadvand $P$, Sunyer J, Basagaña X, Ballester F, Lertxundi A, Fernández-Somoano $A$, et al. 2012. Surrounding greenness and pregnancy outcomes in four Spanish birth cohorts. Environ Health Perspect 120(10):1481-1487, PMID: 22899599, https://doi.org/10.1289/ehp.1205244.

Drouillet P, Forhan A, De Lauzon-Guillain B, Thiébaugeorges 0 , Goua V, Magnin G, et al. 2009. Maternal fatty acid intake and fetal growth: evidence for an association in overweight women. The 'EDEN mother-child' cohort (study of pre- and early postnatal determinants of the child's development and health). Br J Nutr 101(04):575, PMID: 18631416, https://doi.org/10.1017/S0007114508025038.

Duncan DT, Sharifi M, Melly SJ, Marshall R, Sequist TD, Rifas-Shiman SL, et al. 2014. Characteristics of walkable built environments and BMI z-scores in children: evidence from a large electronic health record database. Environ Health Perspect 122(12):1359-1365, PMID: 25248212, https://doi.org/10.1289/ ehp.1307704.

Dzhambov AM, Dimitrova DD, Dimitrakova ED. 2014. Association between residential greenness and birth weight: systematic review and meta-analysis. Urban For Urban Green 13(4):621-629, https://doi.org/10.1016/j.ufug.2014.09.004.

Eeftens M, Beelen R, de Hoogh K, Bellander T, Cesaroni G, Cirach M, et al. 2012. Development of land use regression models for $\mathrm{PM}_{2.5}, \mathrm{PM}_{2.5}$ absorbance, $\mathrm{PM}_{10}$ and $\mathrm{PM}_{\text {coarse }}$ in 20 European study areas; results of the ESCAPE Project. Environ Sci Technol 46(20):11195-11205, PMID: 22963366, https://doi.org/10. 1021/es301948k

European Environment Agency. 2010. Good practice guide on noise exposure and potential health effects. European Commission Working Group Assessment of Exposure to Noise (WG-AEN).
Frank LD, Sallis JF, Conway TL, Chapman JE, Saelens BE, Bachman W. 2006. Many pathways from land use to health: associations between neighborhood walkability and active transportation, body mass index, and air quality. J Am Plan Assoc 72(1):75-87, https://doi.org/10.1080/01944360608976725.

Gascon M, Vrijheid M, Nieuwenhuijsen MJ. 2016. The built environment and child health: an overview of current evidence. Curr Environ Health Rep 3(3):250-257, PMID: 27220615, https://doi.org/10.1007/s40572-016-0094-z.

Grazuleviciene R, Danileviciute A, Nadisauskiene R, Vencloviene J. 2009. Maternal smoking, GSTM1 and GSTT1 polymorphism and susceptibility to adverse pregnancy outcomes. Int J Environ Res Public Health 6(3):1282-1297, PMID: 19440446, https://doi.org/10.3390/ijerph6031282.

Guxens M, Ballester F, Espada M, Fernández M, Grimalt J, Ibarluzea J, et al. 2012. Cohort profile: The INMA -INfancia y Medio Ambiente-(Environment and Childhood) Project. Int J Epidemiol 41(4):930-940, PMID: 21471022, https://doi.org/ 10.1093/ije/dyr054.

Hohmann C, Grabenhenrich L, de Kluizenaar Y, Tischer C, Heinrich J, Chen C-M, et al. 2013. Health effects of chronic noise exposure in pregnancy and childhood: a systematic review initiated by ENRIECO. Int $\mathrm{J}$ Hyg Environ Health 216(3):217-229, PMID: 22854276, https://doi.org/10.1016/j.ijheh.2012.06.001.

Hystad P, Davies HW, Frank L, Van Loon J, Gehring U, Tamburic L, et al. 2014 Residential greenness and birth outcomes: evaluating the influence of spatially correlated built-environment factors. Environ Health Perspect 122(10):10951102, PMID: 25014041, https://doi.org/10.1289/ehp.1308049.

Lamichhane DK, Leem J-H, Lee J-Y, Kim H-C. 2015. A meta-analysis of exposure to particulate matter and adverse birth outcomes. Environ Health Toxicol 30: e2015011, PMID: 26796890, https://doi.org/10.5620/eht.e2015011.

Li M-X, Yeung JMY, Cherny SS, Sham PC. 2012. Evaluating the effective numbers of independent tests and significant $p$-value thresholds in commercial genotyping arrays and public imputation reference datasets. Hum Genet 131(5):747756, PMID: 22143225, https://doi.org/10.1007/s00439-011-1118-2.

Lind PM, Risérus U, Salihovic S, Bavel Bv, Lind L. 2013. An environmental wide association study (EWAS) approach to the metabolic syndrome. Environ Int 55:1-8, PMID: 23454278, https://doi.org/10.1016/j.envint.2013.01.017.

Lioy PJ, Rappaport SM. 2011. Exposure science and the exposome: an opportunity for coherence in the environmental health sciences. Environ Health Perspect 119(11):A466-A467, PMID: 22171373, https://doi.org/10.1289/ehp.1104387.

Magnus P, Irgens LM, Haug K, Nystad W, Skjaerven R, Stoltenberg C. 2006. Cohort profile: The Norwegian Mother and Child Cohort Study (MoBa). Int J Epidemiol 35(5):1146-1150, PMID: 16926217, https://doi.org/10.1093/ije/dyl170.

Maitre L, de Bont J, Casas M, Robinson 0, Aasvang GM, Agier L, et al. 2018. Human Early Life Exposome (HELIX) study: a European population-based exposome cohort. BMJ Open 8(9):0:e021311, PMID: 30206078, https://doi.org/10. 1136/bmjopen-2017-021311.

Mickey RM, Greenland S. 1989. The impact of confounder selection criteria on effect estimation. Am J Epidemiol 129(1):125-137, PMID: 2910056, https://doi.org/ 10.1093/oxfordjournals.aje.a115101.

Ncube CN, Enquobahrie DA, Albert SM, Herrick AL, Burke JG. 2016. Association of neighborhood context with offspring risk of preterm birth and low birthweight: a systematic review and meta-analysis of population-based studies. Soc Sci Med 153:156-164, PMID: 26900890, https://doi.org/10.1016/j.socscimed.2016. 02.014.

Nieuwenhuijsen MJ. 2016. Urban and transport planning, environmental exposures and health - new concepts, methods and tools to improve health in cities. Environ Health 15:S38, PMID: 26960529, https://doi.org/10.1186/s12940016-0108-1.

Nieuwenhuijsen MJ, Dadvand P, Grellier J, Martinez D, Vrijheid M. 2013 Environmental risk factors of pregnancy outcomes: a summary of recent metaanalyses of epidemiological studies. Environ Health 12 Suppl 1:6, PMID: 23320899, https://doi.org/10.1186/1476-069X-12-6.

Nieuwenhuijsen MJ, Khreis H, Triguero-Mas M, Gascon M, Dadvand P. 2017. Fifty shades of green: pathway to healthy urban living. Epidemiology 28(1):63-71, PMID: 27525811, https://doi.org/10.1097/EDE.0000000000000549.

Nieuwenhuijsen MJ, Kruize H, Gidlow C, Andrusaityte S, Antó JM, Basagaña X, et al. 2014. Positive health effects of the natural outdoor environment in typical populations in different regions in Europe (PHENOTYPE): a study programme protocol. BMJ Open 4(4):e004951, PMID: 24740979, https://doi.org/10.1136/ bmjopen-2014-004951.

Nieuwenhuijsen M, Ristovska G, Dadvand P. 2017. WHO environmental noise guidelines for the European region: a systematic review on environmental noise and adverse birth outcomes. Int J Environ Res Public Health 14(10):1252, PMID: 29048350, https://doi.org/10.3390/ijerph14101252.

Patel CJ, Bhattacharya J, Butte AJ. 2010. An Environment-Wide Association Study (EWAS) on type 2 diabetes mellitus. PLoS One 5(5):e10746, PMID: 20505766 https://doi.org/10.1371/journal.pone.0010746.

Patel CJ, Chen R, Kodama K, Ioannidis JP, Butte AJ. 2013a. Systematic identification of interaction effects between genome- and environment-wide ass- 
ociations in type 2 diabetes mellitus. Hum Genet 132(5):495-508, PMID: 23334806, https://doi.org/10.1007/s00439-012-1258-z.

Patel CJ, Manrai AK, Corona E, Kohane IS. 2016. Systematic correlation of environmental exposure and physiological and self-reported behaviour factors with leukocyte telomere length. Int J Epidemiol 46(1):44-56, PMID: 27059547, https://doi.org/10.1093/ije/dyw043.

Patel CJ, Rehkopf DH, Leppert JT, Bortz WM, Cullen MR, Chertow GM, et al. 2013b. Systematic evaluation of environmental and behavioural factors associated with all-cause mortality in the United States National Health and Nutrition Examination Survey. Int J Epidemiol 42(6):1795-1810, PMID: 24345851, https://doi.org/10.1093/ije/dyt208.

Pedersen M, Giorgis-Allemand L, Bernard C, Aguilera I, Andersen AN, Ballester F, et al. 2013. Ambient air pollution and low birthweight: a European cohort study (ESCAPE). Lancet Respir Med 1(9):695-704, PMID: 24429273, https://doi.org/10. 1016/S2213-2600(13)70192-9.

Rahmalia A, Giorgis-Allemand L, Lepeule J, Philippat C, Galineau J, Hulin A, et al. 2012. Pregnancy exposure to atmospheric pollutants and placental weight: an approach relying on a dispersion model. Environ Int 48:47-55, PMID: 22836169, https://doi.org/10.1016/j.envint.2012.06.013.

Robinson 0, Basagaña X, Agier L, de Castro M, Hernandez-Ferrer C, Gonzalez JR, et al. 2015. The pregnancy exposome: multiple environmental exposures in the INMA-Sabadell Birth Cohort. Environ Sci Technol 49(17):10632-10641, PMID: 26168307, https://doi.org/10.1021/acs.est.5b01782.

Robinson 0, Tamayo I, de Castro M, Valentin A, Giorgis-Allemand L, Hjertager Krog N, et al. 2018. The urban exposome during pregnancy and its socioeconomic determinants. Environ Health Perspect 126(7):077005, PMID: 30024382, https://doi.org/10. 1289/EHP2862.

Schembari A, de Hoogh K, Pedersen M, Dadvand P, Martinez D, Hoek G, et al. 2015. Ambient air pollution and newborn size and adiposity at birth: differences by maternal ethnicity (the Born in Bradford Study Cohort.). Environ Health Perspect 123(11):1208-1215, PMID: 25978617, https://doi.org/10.1289/ehp.1408675.

Sinisi SE, van der Laan MJ. 2004. Deletion/Substitution/Addition Algorithms in Learning with Applications in Genomics. Stat Appl Genet Mol Biol 3, PMID: 16646796, https://doi.org/10.2202/1544-6115.1069.

Siroux V, Agier L, Slama R. 2016. The exposome concept: a challenge and a potential driver for environmental health research. Eur Respir Rev 25(140):124-129, PMID: 27246588, https://doi.org/10.1183/16000617.0034-2016.

Slama R, Vrijheid M. 2015. Some Challenges of Studies Aiming to Relate the Exposome to Human Health. Occup Environ Med 72(6):383-384.

Stieb DM, Chen L, Eshoul M, Judek S. 2012. Ambient air pollution, birth weight and preterm birth: a systematic review and meta-analysis. Environ Res 117:100111, PMID: 22726801, https://doi.org/10.1016/j.envres.2012.05.007.

Stingone JA, Buck Louis GM, Nakayama SF, Vermeulen RCH, Kwok RK, Cui Y, et al. 2017. Toward greater implementation of the exposome research paradigm within environmental epidemiology. Annu Rev Public Health 38:315-
327, PMID: 28125387, https://doi.org/10.1146/annurev-publhealth-082516 012750

Stuart EA, Azur M, Frangakis C, Leaf P. 2009. Multiple imputation with large data sets: a case study of the Children's Mental Health Initiative. Am J Epidemiol 169(9):1133-1139, PMID: 19318618, https://doi.org/10.1093/aje/kwp026.

Tzoulaki I, Patel CJ, Okamura T, Chan Q, Brown IJ, Miura K, et al. 2012. A nutrientwide association study on blood pressure. Circulation 126(21):2456-2464 PMID: 23093587, https://doi.org/10.1161/CIRCULATIONAHA.112.114058.

Van Buuren S. 2018. Flexible Imputation of Missing Data. Second Edition. Boca Ration, FL: Chapman \& Hall/CRC.

Vos AA, Posthumus AG, Bonsel GJ, Steegers EAP, Denktas S. 2014. Deprived neighborhoods and adverse perinatal outcome: a systematic review and meta-analysis. Acta Obstet Gynecol Scand 93(8):727-740, PMID: 24834960, https://doi.org/10.1111/ aogs. 12430.

Vrijheid M, Casas M, Gascon M, Valvi D, Nieuwenhuijsen M. 2016. Environmental pollutants and child health - a review of recent concerns. Int J Hyg Environ Health 219(4-5):331-342, PMID: 27216159, https://doi.org/10.1016/j.ijheh.2016.05 001.

Vrijheid M, Slama R, Robinson 0, Chatzi L, Coen M, van den Hazel P, et al. 2014 The Human Early-Life Exposome (HELIX): Project Rationale and Design. Environ Health Perspect 122(6):535-544, PMID: 24610234, https://doi.org/10. 1289/ehp.1307204.

Wang M, Beelen R, Bellander T, Birk M, Cesaroni G, Cirach M, et al. 2014 Performance of multi-city land use regression models for nitrogen dioxide and fine particles. Environ Health Perspect 122(8):843-849, PMID: 24787034, https://doi.org/ 10.1289/ehp.1307271.

Weier J, Herring D. 2000. Measuring Vegetation (NDVI \& EVI). Washington, DC: NASA Earth Observatory. https://earthobservatory.nasa.gov/features/ MeasuringVegetation.

White IR, Royston P, Wood AM. 2011. Multiple imputation using chained equations: issues and guidance for practice. Stat Med 30(4):377-399, PMID: 21225900 https://doi.org/10.1002/sim.4067.

Wild CP. 2005. Complementing the genome with an "exposome": the outstanding challenge of environmental exposure measurement in molecular epidemiology. Cancer Epidemiol Biomarkers Prev 14(8):1847-1850, PMID: 16103423, https://doi.org/10.1158/1055-9965.EPI-05-0456.

Wild CP. 2012. The exposome: from concept to utility. Int J Epidemiol 41(1):24-32, PMID: 22296988, https://doi.org/10.1093/ije/dyr236.

Wright J, Small N, Raynor P, Tuffnell D, Bhopal R, Cameron N, et al. 2013. Cohort profile: The Born in Bradford multi-ethnic family cohort study. Int $\mathrm{J}$ Epidemiol 42(4):978-991, PMID: 23064411, https://doi.org/10.1093/ije/dys112.

Zhang Y, Yu C, Wang L. 2017. Temperature exposure during pregnancy and birth outcomes: an updated systematic review of epidemiological evidence. Environ Pollut 225:700-712, PMID: 28284544, https://doi.org/10.1016/j.envpol. 2017.02.066 